\title{
Comportamento do desempenho neuromuscular após fadiga e resfriamento segundo histórico de prática esportiva
}

\section{Analysis of neuromuscular performance after fatigue and cooling according to a history of sports practice}

Comportamiento del rendimiento neuromuscular tras fatiga y enfriamiento según antecedentes de práctica deportiva

Carlos Alberto Eloy Tavares ${ }^{1}$ Jair José Gaspar Júnior ${ }^{2}$ Ana Paula Anghinoni ${ }^{3}$ Silvio Assis Oliveira-Júnior ${ }^{4}$

${ }^{1}$ Doutor pelo Programa de Pós-Graduação em Saúde e Desenvolvimento na Região Centro-Oeste pela Universidade Federal de Mato Grosso do Sul (UFMS). Docente da Universidade Católica Dom Bosco (UCDB). E-mail: tavares2501@gmail.com, ORCID: http://orcid.org/0000-0002-2173-3591

${ }^{2}$ Mestre pelo Programa de Pós-Graduação em Saúde e Desenvolvimento na Região Centro-Oeste pela Universidade Federal de Mato Grosso do Sul (UFMS). E-mail: gasparjr.ft@gmail.com, ORCID: http://orcid.org/0000-0003-3845-8730

${ }^{3}$ Fisioterapeuta do Programa de Residência Multiprofissional em Cuidados Continuados Integrados (CCl) - Hospital São Julião e Universidade Federal de Mato Grosso do Sul (UFMS). E-mail: anapaula.anghinoni@gmail.com, ORCID: http://orcid.org/0000-0003-0791-6364

${ }^{4}$ Professor associado I da Universidade Federal de Mato Grosso do Sul (UFMS). E-mail: oliveirajr.ufms@gmail.com, ORCID: http://orcid.org/0000-0002-8895-9456 
Resumo: Em geral, diferentes tempos de prática de treinamento podem se associar com alterada resposta neuromuscular em esportistas, durante períodos de fadiga aguda e recuperação pós-esforço. O presente trabalho foi proposto para analisar o desempenho eletromiográfico de músculos extensores do joelho após fadiga e protocolo de resfriamento muscular em universitários com diferentes históricos de prática esportiva. Os participantes $(n=24)$ foram

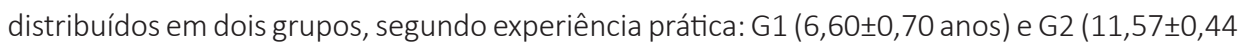
anos); os procedimentos de eletromiografia foram realizados em músculos extensores do joelho durante um exercício isométrico em cadeira extensora. Não foram verificadas diferenças nas comparações entre grupos ( $p>0,05)$. Considerando-se o momento de análise, as medidas de root mean square (RMS) dos músculos vasto lateral e vasto medial foram maiores no momento pósresfriamento, comparado aos momentos inicial e pós-exaustão $(p<0,001)$. Não foram encontradas alterações na força muscular. Em conclusão, o desempenho eletromiográfico de músculos extensores de joelho não é afetado pelo histórico de prática esportiva em atletas universitários.

Palavras-chave: contração isométrica, fadiga muscular, crioterapia, eletromiografia.

Abstract: In general, different training practice times may be associated with altered neuromuscular response in athletes during periods of acute fatigue and in response to post-effort recovery methods. The present investigation was purposed to analyze the electromyographic performance of knee extensor muscles after fatigue and cold-water immersion in university athletes with different practice times. Participants $(n=24)$ were divided into two groups according

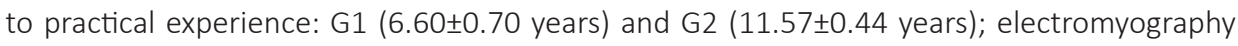
procedures were performed during an isometric knee extensor exercise. There were no differences in the comparisons between groups ( $p>0.05)$. Considering the moment of analysis, root mean square (RMS) measurements of the vastus lateralis and vastus medialis muscle were higher at the post-cryotherapy moment, when compared to the initial and post-exhaustion moments $(p<0.001)$. No changes were observed in relation to muscle strength. In conclusion, neuromuscular performance of knee extensor muscles was not affected by the history of sports practice in university athletes.

Keywords: isometric contraction, muscle fatigue, cryotherapy, electromyography.

Resumen: En general, los diferentes tiempos de la práctica de entrenamiento pueden asociarse con una respuesta neuromuscular alterada en los atletas, durante los períodos de fatiga aguda y recuperación después del esfuerzo. El presente trabajo se propuso analizar el rendimiento electromiográfico de los músculos extensores de la rodilla después de la fatiga y protocolo de enfriamiento muscular en atletas universitarios con diferentes historias de práctica. Los participantes $(n=24)$ fueron distribuidos en dos grupos según

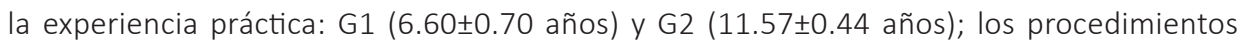
de electromiografía se realizaron en una silla extensora. No hubo diferencias en las comparaciones entre los grupos ( $p>0.05)$. Teniendo en cuenta el momento del análisis, las medidas de la raíz cuadrada media (RMS) del músculo vasto lateral y del músculo vasto medial fueron mayores en el momento posterior a la crioterapia, en comparación con los momentos inicial y posterior al agotamiento $(p<0,001)$. No se encontraron cambios en la fuerza muscular. En conclusión, el rendimiento electromiográfico de los músculos extensores de la rodilla no se ve afectado por la historia de la práctica deportiva en atletas universitarios.

Palabras clave: contracción isométrica, fatiga muscular, crioterapia, electromiografía. 
Comportamento do desempenho neuromuscular após fadiga e resfriamento segundo histórico de prática esportiva

\section{INTRODUÇÃO}

A prática esportiva sistemática e regular tem provocado diferentes consequências em esportistas com históricos variados de experiência prática. Em geral, é comum que atletas ultrapassem os limites de suas condições físicas, o que pode resultar em maior susceptibilidade a lesões, prejudicando o desempenho físico e seus aspectos fisiológicos, assim como a capacidade de recuperação pós-esforço (RPE). Entre as principais condições resultantes da sobrecarga aguda, destaca-se a fadiga muscular (FM) que, em sentido amplo, pode ser descrita como sensação de fraqueza, dor muscular ou diminuição do desempenho durante atividades físicas. Usualmente, a deterioração do desempenho mecânico é o parâmetro que os especialistas adotam para determinar um ponto de fadiga, no qual o músculo não é mais capaz de sustentar um nível de força desejado.

A fadiga é um sintoma comum inespecífico, experimentado por muitos atletas, e está associada a muitas condições de saúde. Muitas vezes, é definida também como uma sensação avassaladora de cansaço, falta de energia e sensação de exaustão, e relaciona-se com uma dificuldade na realização de tarefas voluntárias. O acúmulo de fadiga, se não resolvido, leva a excesso de trabalho, síndrome da fadiga crônica (SFC), síndrome do excesso de treinamento, distúrbios endócrinos, disfunção da imunidade, doenças orgânicas e ameaça à saúde humana. Neste contexto, nas atividades esportivas, quer sejam amadoras, quer sejam profissionais, este tema faz parte da rotina e suas inerentes repercussões fisiológicas acompanham esse público em diversos momentos.

Diante disso, longos períodos de treinamento, bem como o acúmulo de competições com períodos insuficientes aliados a formas inadequadas de recuperação, são geralmente mencionados como fatores que predispõem ao aparecimento de FM. Alguns pesquisadores examinaram a relação entre sinais de eletromiografia (EMG) e FM. Nesse sentido, modelos lineares e não lineares de EMG têm sido utilizados para estimar a FM e alterações neuromusculares em resposta a um período de treinamento.

Os sinais de eletromiografia (EMG) são úteis para entender as atividades musculares, pois estão correlacionadas com as atividades de neurônios 
motores alfa, que representam os comandos motores finais do Sistema Nervoso Central. Esses comandos motores geram contração muscular, o que resulta em tensão muscular. Foi estabelecido que um filtro de passagem baixa de segunda ordem é suficiente para estimar a tensão do músculo por meio de sinais de EMG. Além disso, alguns pesquisadores examinaram a relação entre FM e sinais de EMG. As variáveis frequência média ou frequência mediana, em sinais brutos de EMG, têm sido usadas para estimar a condição de fadiga muscular. Em geral, a redução da frequência mediana, associada com aumento do root mean square (RMS) em sinais brutos de EMG, configura uma característica normalmente utilizada para identificar a FM.

Por sua vez, a eficácia de variados métodos de RPE como complemento a diferentes programas de treinamento, principalmente no esporte competitivo, tem sido objeto de estudo. Nesse aspecto, o resfriamento por crioterapia tem sido amplamente utilizado nos esportes para prevenir a lesão muscular e melhorar a RPE.

Esta estratégia comum de recuperação pós-exercício é implementada regularmente após a prática esportiva e consegue atenuar os efeitos pós-exercícios na capacidade funcional e no desempenho atlético. Embora os mecanismos subjacentes precisos devam ser elucidados, a crioterapia tem a intenção de reduzir a temperatura muscular e atenuar a inflamação.

Portanto, não é surpreendente que a crioterapia demonstre algum efeito sobre a redução dos marcadores de dano oxidativo, provavelmente, por meio de seus efeitos conhecidos, como vasoconstrição, redução na temperatura muscular e atividade inflamatória. Entretanto, não foram encontrados estudos que tenham mostrado os efeitos da crioterapia como método de RPE no desempenho neuromuscular após fadiga aguda em esportistas com diferentes históricos de experiência prática. Estudos que investiguem o tempo de prática esportiva e a relação com desempenho neuromuscular são fundamentais para melhorar o rendimento. A compreensão dos diferentes componentes que contribuem para um alto desempenho numa determinada modalidade esportiva passa pela aplicação de testes específicos que possam auxiliar na elaboração, implementação, avaliação e reformulação de programas de treinamento, bem como no monitoramento dos progressos obtidos. 
Dessa forma, o presente estudo foi proposto para analisar o desempenho neuromuscular de músculos extensores do joelho após indução de fadiga e intervenção com resfriamento muscular em esportistas universitários com diferentes históricos de prática. Em geral, respostas adaptativas derivadas do treinamento regular têm uma relação direta com o tempo de prática esportiva. Nesse contexto, tem-se como hipótese inicial que participantes com maior tempo de experiência prática mostram uma recuperação mais acentuada do desempenho neuromuscular em resposta a fadiga aguda e recuperação por resfriamento.

\section{MÉTODO}

Este estudo tem natureza experimental e a casuística da pesquisa integrou 24 esportistas amadores universitários, com faixa etária entre 18 e 25 anos. Como critérios de inclusão, foi estabelecido ser esportista universitário, do sexo masculino, com prática de treino superior a 12 meses e nenhuma contraindicação para a crioterapia. Foram excluídos aqueles que tivessem histórico de lesão musculoesquelética na atual temporada, atividade laboral caracterizada por importante nível de esforço físico ou que tivessem doença de Raynaud.

Os voluntários assinaram um Termo de Consentimento Livre e Esclarecido (TCLE), concordando com sua participação na pesquisa, que foi aprovada pelo Comitê de Ética em Pesquisa local conforme parecer de número 1.151.455. Os procedimentos de investigação foram desenvolvidos no Laboratório de Estudo do Músculo Estriado (LEME), situado na ClínicaEscola Integrada do Instituto Integrado de Saúde (INISA), da Universidade Federal de Mato Grosso do Sul (UFMS). Todas as coletas de dados foram realizadas em ambiente com temperatura entre $21^{\circ} \mathrm{C}$ e $23^{\circ} \mathrm{C}$ e umidade de $40 \%$ a $60 \%$.

\subsection{Delineamento e procedimentos de estudo}

A caracterização geral foi constituída de anamnese, na qual foram obtidas informações de idade, histórico de treinamento e ocorrência de lesões musculoesqueléticas derivadas da prática esportiva. O registro de lesões 
retrospectivas foi realizado mediante uso do inquérito de morbidade referida (IMR), conforme já realizado em estudos prévios (CAMPOY et al., 2011).

Esse instrumento é adequado para aquisição de informações no formato descritivo, possibilitando fácil interpretação, além de ser direcionado a agravos incidentes, não se restringindo a uma modalidade esportiva específica. Foram tomadas informações sobre ocorrência e prevalência de lesões musculoesqueléticas esportivas na temporada precedente, além de informações sobre natureza, localização anatômica, mecanismos de manifestação, período de afastamento, recorrência, necessidade de acompanhamento médico, entre outros.

Além disso, a caracterização geral integrou avaliação antropométrica, que incluiu as seguintes variáveis: massa corporal, estatura e mensuração de dobras cutâneas. A massa corporal foi aferida com a utilização de uma balança mecânica, com precisão de 0,1 kg e capacidade máxima de 150 kg. Para tanto, os avaliados permaneceram descalços, posicionados em pé, sobre o centro da plataforma da balança e vestindo roupas leves. A mensuração da estatura foi realizada com utilização de um estadiômetro fixo de madeira com precisão de 0,1 cm e extensão máxima de $2 \mathrm{~m}$. Os avaliados permaneceram na posição ortostática, descalços, voltados de costas para a superfície vertical do aparelho e olhando para frente, com os membros superiores relaxados ao lado do tronco, palmas das mãos voltadas para as coxas, calcanhares unidos, tocando a parte vertical do estadiômetro e as bordas mediais afastadas. A parte móvel do estadiômetro foi conduzida até tocar o vértex e comprimir o cabelo.

Para investigar a composição morfológica, foram considerados os perímetros de tórax, cintura, abdômen, quadril, antebraço, coxa e perna com uso de trena antropométrica, marca Sanny, com precisão de 0,1 cm. As espessuras de dobras cutâneas (peitoral, axilar média, subescapular, abdominal, suprailíaca e coxa medial) foram obtidas em triplicata, utilizando-se de um adipômetro. A partir da espessura das dobras cutâneas, foi determinada a densidade corporal (DC).

Ademais, os voluntários foram submetidos à familiarização com os instrumentos, protocolos e procedimentos que foram utilizados na coleta de dados. Para isso, eles foram inicialmente posicionados na cadeira de 
Comportamento do desempenho neuromuscular após fadiga e resfriamento segundo histórico de prática esportiva

teste para reproduzir, sem que houvesse exaustão, os seguintes esforços: contração isométrica voluntária máxima (CIVM, duas repetições com duração de 3 segundos e intervalo de 5 minutos) e contrações submáximas (duas repetições em intensidades correspondentes a 40\% da última CIVM realizada anteriormente, identificada visualmente, com duração de 5 segundos e intervalo de 5 minutos) dos músculos extensores do joelho, para conhecimento e prática em relação aos esforços que foram necessários durante a pesquisa. Nesta ocasião, a ocorrência de exaustão foi evitada por meio do número de repetições, dos tempos de contração e dos intervalos estabelecidos. Foi também apresentada ao voluntário a escala de percepção de esforço que foi utilizada na pesquisa.

Após todos esses esclarecimentos de anamnese e familiarização, perguntou-se ao voluntário sobre a existência de alguma dúvida. Como procedimento-padrão, após intervalo entre cinco e sete dias, outras três etapas ocorreram, com determinação da CIVM e testes de esforço para indução à exaustão, cada uma delas ocorrendo em dias distintos, considerando os intervalos.

Para fins do estudo, os participantes foram distribuídos em dois grupos, tendo por base o histórico de prática esportiva: G1, com praticantes com 1 a 9 anos de treino; e G2, com voluntários com tempo de prática de, pelo menos, 10 anos. Todos os voluntários foram submetidos ao protocolo experimental de duas semanas, que constou de duas etapas: no primeiro dia, foi realizada familiarização, na qual todos os procedimentos de coleta foram explicados e simulados, além de coleta de dados gerais e antropometria, conforme relatado anteriormente.

No segundo dia, realizado na semana seguinte, foi desenvolvida a prova de esforço e resfriamento por imersão em água gelada a 5으 $(\mathrm{Cl} 5$ 으). Para a prova de esforço, foi aferida a força isométrica máxima (FIM), obtida a partir da média de três contrações isométricas voluntárias máximas (CIVM) com duração de 3 segundos e intervalo de 3 minutos entre cada uma. A FIM foi definida pela média dos três maiores valores de força registrados do intervalo de 0,5 segundo em que o voluntário realizava a CIVM.

Todas as contrações foram realizadas numa cadeira extensora feita especialmente para este tipo de teste, com adaptações necessárias para 
acomodação dos voluntários. Os sujeitos permaneceram na postura sentada sobre a cadeira, contidos na altura do tronco e das coxas para estabilização, com quadril e joelho flexionados a 90․ Os esforços isométricos foram realizados com o membro inferior dominante, no sentido de estender a perna, e os valores de força foram obtidos a partir de uma célula de carga. Houve sempre o estímulo verbal para que o esforço fosse o mais extenuante possível. Esta etapa foi descrita como pré-exaustão (T1).

Em seguida, os participantes foram submetidos ao teste submáximo de exaustão (indução de fadiga), a 40\% da FIM. A fadiga foi determinada a partir de duas situações: encerramento voluntário do teste ou impossibilidade de manutenção do nível de esforço em até $5 \%$ do valor proposto durante 3 segundos. Imediatamente após o teste submáximo de exaustão, foi realizada nova CIVM, etapa esta denominada de pós-exaustão (T2). Antes de executarem a CIVM em T1, os voluntários permaneceram 5 minutos em repouso. Após o teste de exaustão e posterior CIVM, os atletas foram submetidos à imersão a $5^{\circ} \mathrm{C}$ por 10 minutos. Para tanto, foi adotada postura ortostática com membros inferiores imersos em água resfriada por cubos de gelo em um tonel com dimensões de $980 \mathrm{~mm}$ de altura, $490 \mathrm{~mm}$ de largura, 560 mm de comprimento e capacidade para 240 litros d'água.

Por fim, todos realizaram mais uma CIVM para verificar o efeito do resfriamento muscular, denominada pós-crioterapia (T3). Durante todas as contrações, foi dado estímulo verbal aos voluntários.

\subsection{Eletromiografia de superfície}

Para a obtenção da atividade eletromiográfica, foi utilizado um eletromiógrafo da marca Miotec (Miotec Equipamentos Biomédicos LTDA, Porto Alegre, RS, Brasil) de quatro canais, calibrado com um ganho de 1.000 vezes, modo comum de rejeição de $80 \mathrm{~dB}$, frequência de amostragem de $2.000 \mathrm{~Hz}$, filtro passa-alta de $10 \mathrm{~Hz}$ e filtro passa-baixa de $500 \mathrm{~Hz}$. A EMG dos músculos vasto lateral (VL), reto femoral (RF) e vasto medial (VM) foi captada durante os procedimentos de CIVM e de exaustão.

Foram utilizados eletrodos de superfície de $\mathrm{Ag} / \mathrm{AgCl}$ (modelo MediTrace, marca Kendal, Chicopee, MA, United States, USA), posicionados 
Comportamento do desempenho neuromuscular após fadiga e resfriamento segundo histórico de prática esportiva

conforme recomendação internacional da Surface Electromyograhpy for the Non-Invasive Assessment of Muscles (SENIAM). A distância entre eletrodos foi de $3 \mathrm{~cm}$ e um eletrodo de referência foi posicionado sobre o maléolo fibular.

Previamente à colocação dos eletrodos, foram realizados procedimentos para minimizar interferências no sinal eletromiográfico, entre eles, a tricotomia das regiões em que foram colocados os eletrodos para otimizar a penetração do estímulo elétrico, além de posterior assepsia para retirada de oleosidade da pele. Para o vasto lateral, os eletrodos foram posicionados a 2/3 da linha da espinha ilíaca anterior superior até linha articular lateral da patela. Para o reto femural, os eletrodos foram colocados a $50 \%$ da linha da espinha ilíaca ântero-superior até a parte superior da patela. Para o vasto medial, os eletrodos foram posicionados a $80 \%$ da distância entre a espinha ilíaca anterior superior e o espaço articular em frente à borda anterior do ligamento medial.

\subsection{Análise estatística}

Os dados são apresentados de forma descritiva, em média e erro-padrão. As características gerais, em valores paramétricos e não paramétricos, foram analisadas com emprego de teste $t$ de Student e teste de Mann-Whitney, respectivamente. Para a análise dos resultados de força e RMS, considerando-se a combinação entre grupos e períodos de análise, foi utilizada análise de variância de duas vias, no modelo de medidas repetidas (Two-Way RM ANOVA), e teste de Bonferroni.

\section{RESULTADOS}

Na Tabela 1, são apresentadas as características descritivas dos grupos estudados. A idade dos participantes de ambos os grupos variou entre 18 e 25 anos, sem diferença significativa entre os grupos $(p=0,60)$. Situações similares ocorreram com massa corporal $(p=0,78)$ e estatura $(p=0,84)$. 0 tempo de prática esportiva de G1 variou entre 2 e 9 anos, sendo significativamente menor do que em G2, que variou entre 10 e 14 anos $(p<0001)$. 
Tabela 1 - Características gerais dos participantes, segundo grupo de estudo

\begin{tabular}{lccc}
\hline \multirow{2}{*}{ Características } & \multicolumn{2}{c}{ Grupos } & \multirow{2}{*}{ Valor de $\mathbf{p}$} \\
\cline { 2 - 3 } & $\mathbf{G 1} \mathbf{( n = 1 0 )}$ & $\mathbf{G 2}(\mathbf{n}=\mathbf{1 4})$ & \\
\hline Idade (anos) & $21,10 \pm 0,72$ & $21,57 \pm 0,56$ & $0,60^{(\mathrm{a})}$ \\
Massa $(\mathrm{kg})$ & $81,37 \pm 2,89$ & $82,74 \pm 3,61$ & $0,78^{(\mathrm{a})}$ \\
Estatura $(\mathrm{m})$ & $1,84 \pm 0,02$ & $1,78 \pm 0,02$ & $0,84^{(\mathrm{b})}$ \\
Tempo de Prática (anos) & $6,60 \pm 0,70$ & $11,57 \pm 0,44$ & $<0,0001^{*(\mathrm{~b})}$ \\
\hline
\end{tabular}

Variáveis massa corporal e estatura são apresentadas em média e desvio-padrão; demais valores, expressos em médias e erro-padrão; ${ }^{(a)} T e s t e ~ t$ de Student; ${ }^{(b)}$ Teste Mann-Whitney; *diferença estatisticamente significativa entre grupos.

Em relação à força muscular, os grupos mostraram comportamento similar ao longo do tempo de análise $(p>0,05)$. Considerando-se os efeitos do momento de avaliação, os índices de força (kgf) foram maiores em T1 em comparação a T2 $(p<0,05)$. Em T3, não foram constatadas diferenças nos níveis de força, quando comparado a T1 e T2 (Tabela 2).

Tabela 2 - Medidas de força (kgf), segundo grupo e momento

\begin{tabular}{ccccccc}
\hline \multirow{2}{*}{ Grupo } & \multicolumn{3}{c}{ Momento de análise } & \multicolumn{3}{c}{ p-valor } \\
\cline { 2 - 6 } & T1 & T2 & T3 & Grupo & Momento & Interação \\
\hline G1 & $62,2 \pm 5,4^{\mathrm{Ba}}$ & $49,3 \pm 3,7^{\mathrm{Aa}}$ & $55,4 \pm 3,7^{\mathrm{ABa}}$ & \multirow{2}{*}{0,607} & $<0,001$ & \multirow{2}{*}{0,383} \\
\hline G2 & $60,5 \pm 3,5^{\mathrm{Ba}}$ & $53,0 \pm 3,2^{\mathrm{Aa}}$ & $58,6 \pm 2,9^{\mathrm{ABa}}$ & & & \\
\hline
\end{tabular}

Valores expressos em média \pm erro-padrão. A, B Letras maiúsculas distintas indicam diferenças estatisticamente significativas $(p<0,05)$ entre momentos dentro do grupo; $a, b$ Letras minúsculas distintas indicam diferenças estatisticamente significativas $(p<0,05)$ entre grupos dentro do momento; Two-Way RM ANOVA e teste de Bonferroni.

Em relação ao RMS, foram identificadas diferenças entre os tempos de análise do músculo vasto lateral (VL) nos atletas de $G 2(p<0,001)$, sendo T3 significativamente maior do que as aferições em T1 e T2 $(p<0,05)$. Comportamento similar foi observado na comparação entre os tempos de análise do músculo vasto medial (VM) também para o G2, sendo o momento pós-crioterapia (T3) significativamente maior do que T1 e T2 ( $<<0,05)$. Os grupos se comportaram de forma semelhante em relação ao RMS nos três músculos avaliados e nos três tempos de teste $(p<0,05)$, conforme Tabela 3. 
Comportamento do desempenho neuromuscular após fadiga e resfriamento segundo histórico de prática esportiva

Tabela 3 - Medidas de root mean square (RMS), segundo grupo e momento

\begin{tabular}{|c|c|c|c|c|c|c|c|}
\hline \multirow[b]{2}{*}{ Músculo } & \multirow[b]{2}{*}{ Grupo } & \multicolumn{3}{|c|}{ Momento de análise } & \multicolumn{3}{|c|}{ p-valor } \\
\hline & & T1 & T2 & T3 & Grupo & $\begin{array}{c}\text { Mo- } \\
\text { mento }\end{array}$ & $\begin{array}{c}\text { Intera- } \\
\text { ção }\end{array}$ \\
\hline \multirow{2}{*}{ VL } & G1 & $469,5 \pm 47,1^{\mathrm{ABa}}$ & $431,0 \pm 44,1^{\mathrm{Aa}}$ & $513,2 \pm 65,1^{\mathrm{Ba}}$ & \multirow{2}{*}{0,742} & \multirow{2}{*}{$<0,001$} & \multirow{2}{*}{0,244} \\
\hline & $\mathrm{G} 2$ & $467,3 \pm 45,4^{\mathrm{Aa}}$ & $432,6 \pm 29,6^{\mathrm{Aa}}$ & $574,3 \pm 43,9^{\mathrm{Ba}}$ & & & \\
\hline \multirow{2}{*}{ RF } & G1 & $504,7 \pm 71,8^{\mathrm{Aa}}$ & $466,1 \pm 71,6^{\mathrm{Aa}}$ & $527,5 \pm 51,7^{\text {Аa }}$ & \multirow{2}{*}{0,845} & \multirow{2}{*}{0,047} & \multirow{2}{*}{0,618} \\
\hline & $\mathrm{G} 2$ & $487,9 \pm 41,2^{\mathrm{Aa}}$ & $487,6 \pm 38,3^{\mathrm{Aa}}$ & $563,3 \pm 48,9^{\mathrm{Aa}}$ & & & \\
\hline \multirow{2}{*}{ VM } & G1 & $439,1 \pm 53,4^{\mathrm{Aa}}$ & $442,1 \pm 27,4^{\mathrm{ABa}}$ & $529,9 \pm 58,9^{\mathrm{Ba}}$ & \multirow{2}{*}{0,483} & \multirow{2}{*}{$<0,001$} & \multirow{2}{*}{0,902} \\
\hline & G2 & $495,7 \pm 46,7^{\mathrm{Aa}}$ & $477,5 \pm 47,0^{A a}$ & $572,5 \pm 45,4^{\mathrm{Ba}}$ & & & \\
\hline
\end{tabular}

Valores expressos em média \pm erro-padrão. A, B Letras maiúsculas distintas indicam diferenças estatisticamente significativas $(p<0,05)$ entre momentos dentro do grupo; $a, b$ Letras minúsculas distintas indicam diferenças estatisticamente significativas $(p<0,05)$ entre grupos dentro do momento; Two-Way RM ANOVA e teste de Bonferroni.

\section{DISCUSSÃO}

No estudo da fadiga muscular, destaca-se a participação de componentes centrais e periféricos (ALMEIDA et al., 2011). A força muscular é afetada pelos componentes periféricos como tipo e intensidade do exercício, grupos musculares envolvidos, e ambiente bioquímico e físico local. Este aspecto evidencia a fadiga muscular como um processo complexo e dotado de inúmeras variáveis que envolvem aspectos fisiológicos, biomecânicos e psicológicos. São diversos os tipos de fadiga muscular e o quanto cada um contribui para a diminuição da performance muscular estabelece relação com o tipo de fibra, intensidade e duração da atividade física (BILLAUT et al. 2006).

As pesquisas envolvendo lesões esportivas estão sempre associadas a mudanças na potência, resistência, trabalho, torque e desempenho muscular (KANNUS et al., 2003). O acompanhamento das taxas metabólicas durante o exercício e gasto energético dos músculos é fundamental para entendimento do desempenho muscular. Estudos apontaram que jogadores de futebol precisam de pelo menos 72 horas para recuperar totalmente os músculos submetidos a estresse oxidativo, imposto por uma partida de futebol. A compreensão das causas da fadiga muscular faz parte de uma temática que, apesar de todos os estudos, ainda apresenta controvérsia por se tratar de um conceito multidimensional, que abrange aspectos fisiológicos e psicológicos; 
quantificar os sintomas que precedem a perda na capacidade do músculo em sustentar um nível de força caracteriza uma situação complexa no meio clínico e esportivo (ZWARTS; BLEIJENBERG; VAN ENGELEN, 2008).

No esporte de elite, tanto a carga de treinamento quanto os períodos de recuperação são observados, considerando distúrbios transitórios na variação da força muscular para posterior acompanhamento e gestão de desempenho (FISKERSTRAND; SEILER, 2004). Esta gestão tem por objetivo evitar a fadiga, a qual poderia provocar diminuição do desempenho. Tal como descrito por Meeusen et al. (2013), a fadiga segue um processo contínuo que vai desde a fadiga voluntária e controlada, necessária para a progressão do desempenho e que solicita algumas horas ou alguns dias de recuperação, até a involuntária, que demanda semanas ou mesmo meses de recuperação, típica da condição de overtraining (OT).

O presente estudo teve como objetivo analisar o desempenho eletromiográfico de três músculos do quadríceps submetidos à fadiga e ao protocolo de resfriamento muscular em esportistas universitários com diferentes históricos de prática. Para tanto, a fadiga muscular foi monitorada a partir de sinais EMG. Em particular, monitoramos um parâmetro que caracteriza a fadiga muscular com base na frequência e magnitude dos sinais EMG. Em seguida, verificamos a eficácia desse parâmetro na avaliação quantitativa da fadiga muscular. Segundo a hipótese inicial, participantes com maior tempo de experiência prática teriam uma recuperação mais breve do desempenho neuromuscular após fadiga aguda e recuperação por resfriamento. Contudo, os grupos não mostraram diferenças nas variáveis investigadas, comprovando que o histórico de treinamento não está associado com diferenças no rendimento eletromiográfico pós-esforço.

Por sua vez, foram verificados maiores valores de força no início (T1), em comparação aos tempos pós-exaustão e pós-crioterapia (T2 e T3) nos dois grupos. Portanto, no que diz respeito à ativação voluntária máxima inalterada, este resultado confirma que o protocolo foi eficaz na indução de fadiga periférica local substancial dos músculos extensores do joelho, com efeitos negligenciáveis dos mecanismos centrais, semelhante a estudos anteriores (SILVA, 2008). 
Comportamento do desempenho neuromuscular após fadiga e resfriamento segundo histórico de prática esportiva

As médias de força em que foi iniciado o experimento, tanto para G1 como para o G2, foram muito semelhantes, o que fortalece a homogeneidade dos voluntários neste quesito. Evidente decréscimo de 20,84\% em G1 e 15,21\% em G2 apareceram quando se comparam os valores de T1 com T2, confirmando a fadiga, e foi observado que T3 (pós-crioterapia) em relação a T1 (pré-exaustão) mostrou recuperação parcial de 12,33\% para o G1 e 3,23\% para G2, porém, sem significância estatística.

Esses achados estão de acordo com estudos prévios utilizando contrações isométricas para induzir a fadiga, os quais documentaram diminuição na força voluntária máxima por 30 minutos após o protocolo de fadiga (Ll et al., 2016). Isso demonstra que a fadiga ocorreu ao longo de todo o protocolo e, portanto, que as mudanças na coordenação motora relatada se originaram da fadiga periférica do quadríceps unilateral (POTVIN; FUGLEVAND, 2017).

O comportamento do valor de RMS nos músculos analisados de G1 e G2 foi semelhante, com redução de T1 para T2 nos três músculos, sendo de 8,20\% (VL), 7,66\% (RF) e 0,7\% (VM). De T2 para T3, houve aumento de, respectivamente, $19,1 \%, 13,2 \%$ e $19,9 \%$ para os mesmos músculos e sequências anteriores em G1. Para G2, o comportamento apresentou redução de T1 para T2 de 7,1\% (VL), 0,1\% (RF) e 3,7\% (VM), com aumento de T2 para T3 de 32,8\% (VL), 15,5\% (RF) e 19,9\% (VM), em conformidade com estudo que analisou o sinal de RMS no domínio do tempo e conseguiu quantificar o tempo no qual o músculo ficou ativo (KURIKI, 2012).

Vale destacar que, quando se comparou T3 em relação a T1 para G1, todos os músculos apresentaram valores de RMS maiores em relação ao tempo inicial em que foram realizadas as primeiras CIVMs, verificando-se aumento de 9,2\% (VL), 4,4\% (RF) e 6,9\% (VM). Para G2, houve aumento de 22,9\% (VL), 15,4\% (RF) e 15,5\% (VM). Quando analisamos o RMS em momentos isolados, não há um padrão de aumento, que é o comum quando está ocorrendo fadiga muscular, mesmo nos momentos em que a coleta foi realizada imediatamente uma após a outra, como de T1 para T2. De T3 para T1, houve aumento de RMS, demonstrando possível resposta à recuperação.

Uma vez verificado, neste estudo, que o tempo de prática esportiva pouco interferiu na qualidade da recuperação da fadiga, voltamo-nos de modo especial para o efeito fisiológico da crioterapia por imersão a 5 으 
neste processo. A crioterapia tem sido amplamente utilizada nos esportes para prevenir a lesão muscular e melhorar a recuperação (LEEDER et al., 2012). Nesse sentido, apesar da limitada repercussão no aumento da FIM, a crioterapia mostrou algum efeito de recuperação na percepção subjetiva da FIM, provavelmente, por meio de seus efeitos conhecidos na vasoconstrição, redução na temperatura muscular e atividade inflamatória (POURNOT et al., 2011). Isso implica que o dano oxidativo gerado pelo processo de isquemia-reperfusão pode ter sido reduzido.

O uso de imersão em água fria a temperaturas $<15^{\circ} \mathrm{C}$ é uma estratégia cada vez mais popular de recuperação. Tem sido proposto que a pressão hidrostática exercida sobre o corpo, quando imerso em água fria, provoca mudanças intracelulares de fluido, de forma a reduzir a inflamação e o edema, preservando, assim, a função muscular e a performance muscular (WILCOCK, 2006).

Estudos anteriores relatam que a crioterapia pode reduzir a velocidade de condução nervosa, não apenas alterando a percepção da dor, mas também interferindo no recrutamento de unidades motoras (GARCÍA-MANSO et al., 2011). Há um conjunto emergente das provas que sustentam o uso de imersão em água fria entre séries repetidas de exercícios de alta intensidade, ao longo de vários dias. Em um estudo investigando os efeitos de imersão em água fria durante um torneio de futebol de 4 dias, a imersão em água fria atenuou decréscimos na performance de corrida (ROWSELL et al., 2011).

Em outro estudo sobre jogadores de basquete, imersão em água fria melhorou o desempenho do salto (DELEXTRAT et al., 2013). Em ambos os estudos, imersão em água fria levou a reduzir a percepção de fadiga geral e dor no dia seguinte, em comparação com outras intervenções de recuperação. Além disso, a imersão em água fria imediatamente após uma sessão de treinamento de alta intensidade resulta em melhor desempenho de execução no dia seguinte, quando se compara com atraso na imersão em água fria realizada três horas pós-exercício (BROPHY-WILLIAMS; LANDERS; WALLMAN, 2011).

Muitos esportes de equipe usam imersão em água fria durante o intervalo da competição como uma estratégia para melhorar a performance na sequência do jogo ou competição (ROWSELL et al., 2011). Por isso, 
Comportamento do desempenho neuromuscular após fadiga e resfriamento segundo histórico de prática esportiva

estudos envolvendo a fadiga muscular são uma importante ferramenta terapêutica para avaliar o impacto da imersão em água fria na oxigenação muscular durante o desempenho do exercício subsequente. O resfriamento é utilizado também como estratégia de recuperação entre jogos ou competições em dias alternados, para verificar os seus efeitos após horas ou dias de uso da crioterapia, porém, neste estudo, objetivamos verificar os efeitos na recuperação muscular durante a atividade esportiva em atletas com diferentes tempos de experiência em ambientes competitivos, ou seja, entre um intervalo de uma luta em relação à outra ou mesmo no intervalo de uma partida de futebol, por exemplo.

Há necessidade de que futuros estudos sejam desenvolvidos para investigar a eficácia de outros protocolos de crioterapia, considerando-se parâmetros de temperatura e duração. Da mesma forma, não se deve descartar que outras referências de experiência prática, como nas faixas de 1 a 5 anos e acima de 15 anos de histórico de treinamento, possam repercutir em alterações adaptativas no contexto pós-esforço e mereçam ser mais bem estudadas.

\section{CONCLUSÃO}

Esportistas com diferentes históricos de experiência prática mostram desempenho neuromuscular similar após condição de fadiga aguda e recuperação por resfriamento.

\section{FONTES DE FINANCIAMENTO}

Este estudo contou com apoio financeiro da Universidade Federal de Mato Grosso do Sul (UFMS), Conselho Nacional de Desenvolvimento Científico e Tecnológico (CNPq) e Coordenação de Aperfeiçoamento de Pessoal de Nível Superior (CAPES) - código de financiamento 001.

\section{REFERÊNCIAS}

ALMEIDA, P.; LOPES-MARTINS, R. A. B.; MARCHI, T.; TOMAZONI, S. S.; ALBERTINI, R.; CORRÊA, J. C. F.; ROSSI, R. P.; MACHADO, G. P.; SILVA, D. P.; BJORDAL, J. M.; LEAL 
JUNIOR, E. C. P. Red (660 nm) and infrared (830 nm) low-level laser therapy in skeletal muscle fatigue in humans: what is better? Lasers Medical Science, Berlim, v. 27, n. 2, p. 453-58, 2011.

BILLAUT, F.; BASSET, F. A.; GIACOMONI, M.; LEMAITRE, F.; TRICOT, V.; FALGAIRETTE G. Effect of high-intensity intermittent cycling sprints on neuromuscular activity. International Journal of Sports Medicine, Nova York, v. 27, p. 25-30, 2006.

BROPHY-WILLIAMS, N.; LANDERS, G.; WALLMAN, K. Effect of immediate and delayed cold water immersion after a high intensity exercise session on subsequent run performance. Journal of Sports Science \& Medicine, Abingdon, v. 10, p. 665-70, 2011.

CAMPOY, F. A.; COELHO L. R.; BASTOS, F. N.; NETTO JUNIOR, J.; VANDERLEI, L. C.; MONTEIRO, H. L.; PADOVANI, C. R.; PASTRE, C. M. Investigation of risk factors and characteristics of dance injuries. Clinical Journal of Sport Medicine: official journal of the Canadian Academy of Sport Medicine, Alphen aan den Rijn, v. 21, n. 6, p. 493-8, 2011.

DELEXTRAT, A.; CALLEJA-GONZALEZ, J.; HIPPOCRATE, A.; CLARKE, N. D. Effects of sports massage and intermittent cold-water immersion on recovery from matches by basketball players. Journal of Sports Sciences, Londres, v. 31, p. 11-19, 2013.

FISKERSTRAND, A.; SEILER, K. S. Training and performance characteristics among Norwegian international rowers 1970-2001. Scandinavian Journal of Medicine \& Science in Sports, Hoboken, v. 14, p. 303-10, 2004.

GARCÍA-MANSO, J. M.; RODRÍGUEZ-MATOSO, D.; RODRÍGUEZ-RUIZ, D.; SARMIENTO, S.; DE SAA, Y.; CALDERÓN, J. Effect of cold-water immersion on skeletal muscle contractile properties in soccer players. American Journal of Physical Medicine \& Rehabilitation, Alphen aan den Rijn, n. 90, p. 356-63, 2011.

GONZALEZ-IZAL, M.; MALANDA, A.; NAVARRO-AMÉZQUETA, I.; GOROSTIAGA, E. M.; MALLOR, F.; IBAÑEZ, J.; IZQUIERDO, M. EMG spectral indices and muscle power fatigue during dynamic contractions. Journal of Electromyography and Kinesiology, Oxford, v. 20, n. 2, p. 233-40, 2010.

KANNUS, P.; PARKARRI, J.; JÄRVINEN, T. L.; JÄRVINEN, T. A.; JÄRVINEN, M. Basic science and clinical studies coincide: active treatment approach is needed after a sports injury. Scandinavian Journal of Medicine \& Science in Sports, Hoboken, n. 13, p. 150-4, 2003. 
Comportamento do desempenho neuromuscular após fadiga e resfriamento segundo histórico de prática esportiva

KURIKI, H. U; AZEVEDO, F. M. The relationship between electromyography and muscle force. In: SCHWARTZ, M. (Ed.). EMG Methods for Evaluating Muscle and Nerve Function. InTech, 2012. p. 31-54.

LEE, K. A.; HICKS, G.; NINO-MURCIA, G. Validity and reliability of a scale to assess fatigue. Psychiatry Research, Amsterdã, Países Baixos, v. 36, n. 3, p. 291-8, 1991.

LEEDER, J.; GISSANE, C.; VAN SOMEREN, K.; GREGSON, W.; HOWATSON, G. Cold water immersion and recovery from strenuous exercise: a meta-analysis. British Journal of Sports Medicine, Doncaster, Reino Unido, v. 46, p. 233-40, 2012.

LI, L.; SHIN, H.; LI, X.; LI, S.; ZHOU, P. Localized electrical impedance myography of the biceps brachii muscle during different levels of isometric contraction and fatigue. Sensors, Basel, v. 16, n. 4, p. 1-11, 2016. doi: 10.3390/s16040581

MEEUSEN, R.; DUCLOS, M.; FOSTER, C.; FRY, A.; GLEESON, M.; NIEMAN, D.; RAGLIN, J.; RIETJENS, G.; STEINACKER, J.; URHAUSEN, A.; EUROPEAN COLLEGE OF SPORT SCIENCE; AMERICAN COLLEGE OF SPORTS MEDICINE. Prevention, diagnosis, and treatment of the overtraining syndrome: joint consensus statement of the European College of Sport Science and the American College of Sports Medicine. Medicine and Science in Sports and Exercise, Filadélfia, n. 45, p. 186-205, 2013.

POTVIN, J. R.; FUGLEVAND, A. J. A motor unit-based model of muscle fatigue. PLoS Computational Biology; São Francisco, v. 13, n. 6, e1005581, 2017 doi:10.1371/ journal.pcbi.1005581

POURNOT, H.; BIEUZEN, F.; DUFFIELD, R.; LEPRETRE, P. M.; COZZOLINO, C.; HAUSSWIRTH, C. Short term effects of various water immersions on recovery from exhaustive intermittent exercise. European Journal of Applied Physiology, Basingstoke, n. 111, v. 7, p. 1287-95, 2011.

ROGERS, D. R.; MACISAAC, D. T. Training a multivariable myoelectric mapping function to estimate fatigue. Journal of Electromyography and Kinesiology, Oxford, v. 20, n. 5, p. 953-60, 2010.

ROWSELL, G. J.; COUTTS, A. J.; REABURN, P.; HILL-HAAS. S. Effect of post-match coldwater immersion on subsequent match running performance in junior soccer players during tournament play. Journal of Sports Sciences, Londres, n. 29, p. 1-6, 2011.

SANTOS, C. C.; PEREIRA, L. S. M.; RESENDE, M. A., MAGNO, F.; AGUIAR, V. Aplicação da versão brasileira do questionário de dor McGILL em idosos com dor crônica. Acta Fisiatrica., São Paulo, v. 13, p. 75-82, 2006. 
SILVA, S. R. D. DA; GONÇALVES, M. Análise da fadiga muscular pela amplitude do sinal eletromiográfico. Revista Brasileira de Ciência e Movimento, Brasília, v. 11, n. 3, p. 15-20, 2008.

SOO, Y.; SUGI, M.; YOKOI, H.; ARAI, T.; NISHINO, M.; KATO, R.; NAKAMURA, T.; OTA, $J$. Estimation of handgrip force using frequency-band technique during fatiguing muscle contraction. Journal of Electromyography and Kinesiology, Oxford, v. 20, n. 5, p. 888-95, 2010.

WILCOCK, I. M.; CRONIM, J. B.; HING, W. A. Physiological response to water immersion: a method for sport recovery? Sports Medicine, Auckland, v. 36, n. 9, p. 747-65, 2006.

ZWARTS, M. J., G. BLEIJENBERG, AND B. G. M. VAN ENGELEN, Clinical neurophysiology of fatigue. Clinical Neurophysiology, Amsterdã, v. 119, n. 1, p. 2-10, 2008. 